\title{
POBREZA E GÊNERO: EVOLUÇÃO DOS INDICADORES DE POBREZA FOSTER-GREER-THORBECKE (FGT) PARA
}

\section{O BRASIL}

\author{
Poverty and gender: Evolution of poverty indicators Foster-Greer-Thorbecke \\ (FGT) for Brazil
}

\author{
Chinara Mendes SCHINAIDER \\ Departamento de Economia \\ Universidade Federal de Viçosa, (UFV), Minas Gerais, Brasil \\ chinaraschinaider@hotmail.com \\ Izabella Maria da Silva VIANA \\ Departamento de Economia \\ Universidade Federal de Viçosa, (UFV), Minas Gerais, Brasil \\ izabellam29@gmail.com \\ Evandro Camargos TEIXEIRA \\ Departamento de Economia \\ Universidade Federal de Viçosa, (UFV), Minas Gerais, Brasil \\ evandro.teixeira@ufv.br
}

\begin{abstract}
RESUMO
Objetivo: O objetivo desse artigo é avaliar a hipótese de que houve feminização da pobreza no Brasil nos anos de 2005 e 2015, utilizando-se dados da Pesquisa Nacional por Amostra de Domićlios do Instituto Brasileiro de Geografia e Estatística (IBGE). A partir do índice de pobreza Foster-Greer-Thorbecke (FGT), foi calculada a proporção de pobres (P0) em relação ao restante da população, o hiato de pobreza $(\mathrm{P} 1)$ e sua severidade $(\mathrm{P} 2)$ para o Brasil e suas macrorregiões, segundo gênero, posição na ocupação e na família. Os principais resultados não apontam evidências de feminização da pobreza no período quando são comparados os gêneros. Porém, levando-se em consideração a posição de ocupação da mulher (chefe ou cônjuge) e o tipo de família (mulher com filhos), houve aumento na incidência e intensidade da pobreza ao longo dos anos analisados.
\end{abstract}

PALAVRAS-CHAVE: Pobreza. Gênero. Economia feminista.

\begin{abstract}
Objetive: The objective of this article is to evaluate the hypothesis that there was a feminization of poverty in Brazil in the years 2005 and 2015, using data from the National Household Sample Survey of the Brazilian Institute of Geography and Statistics (IBGE). From the Foster-Greer-Thorbecke poverty index (FGT), the proportion of poor (P0) in relation to the rest of the population was calculated, the poverty gap (P1) and its severity (P2) for Brazil and its macro-regions, according to gender, position in occupation and family. The main results do not point to evidence of feminization of poverty in the period when genders are compared. However, taking into account the position of occupation of the woman (boss or spouse) and the type of family (woman with children), there was an increase in the incidence and intensity of poverty over the years analyzed.

KEYWORDS: Poverty. Gender. Feminist economics.
\end{abstract}

Classificação JEL: B54; B55; 132

Recebido em: 01-03-2020. Aceito em: 06-07-2020. 


\section{INTRODUÇÃO}

Pode-se definir pobreza como algum tipo de privação, que pode estar relacionada a bens materiais ou incluir elementos de ordem cultural e social, frente a recursos disponíveis de uma pessoa ou família (KAGEYAMA e HOFFMANN, 2006).

Em estudo realizado pela OXFAM (2017), a pobreza estava presente na vida de mais de 700 milhões de pessoas no mundo, tratando-se de uma situação extrema. No Brasil, muitos trabalhos já foram realizados retratando esta temática, entretanto aqueles que relacionam pobreza e gênero ainda são escassos. Especificamente, no que tange esta relação, a visão majoritariamente aceita nos últimos anos é de que vem ocorrendo empobrecimento mais acentuado das mulheres ou das pessoas em domicílios chefiados por mulheres. Em consequência, esses dois grupos estariam entre os mais pobres, mas este não é consenso na literatura (COSTA et al., 2005).

Tal expectativa denotada pela literatura advém das estatísticas recentes. De acordo com o Relatório do Desenvolvimento Humano (1995), a pobreza tem perfil feminino - de 1,3 bilhões de pessoas consideradas pobres, $70 \%$ são mulheres. Esta proporção, no entanto, é questionada por Marcoux (1998), pois se $70 \%$ dos pobres são mulheres, então ter-se-ia a proporção de 4,3 mulheres pobres para cada homem pobre (NOVELLINO, 2004). Dada a heterogeneidade entre as regiões, a hipótese de que há feminização da pobreza não pode ser generalizada, pois o acesso ao mercado de trabalho e educação são distribuídos de maneira desigual, assim como as relações monoparentais.

Levando em consideração o que fora destacado, o objetivo da presente pesquisa é avaliar a hipótese de que houve feminização da pobreza no Brasil, comparando-se os anos de 2005 e 2015. Para fins de análise, neste estudo, o fenômeno de feminização da pobreza ocorrerá quando a pobreza apresentar crescimento (absoluto ou relativo) entre as mulheres. Nesse sentido, a pobreza feminina será confirmada, em termos absolutos, quando houver crescimento dos níveis de pobreza para as mulheres; e em termos relativos, se o grupo das mulheres apresentar redução dos níveis de pobreza em menor grau em relação à redução entre os homens - considerando o mesmo para mulheres chefes, mulheres cônjuges e famílias chefiadas por mulheres.

O estudo está dividido em cinco seções. Além dessa introdução, são apresentados na segunda seção estudos que debatem a temática da pobreza relacionada ao gênero e as principais definições sobre feminização da pobreza. Posteriormente, na terceira seção é 
abordada a metodologia adotada. Na quarta seção, são analisados os resultados. Por fim, na última seção, são apresentadas as considerações finais.

\section{POBREZA E GÊNERO: QUESTÕES TEÓRICAS E EMPÍRICAS}

Embora existam diversos estudos que denotam aumento da pobreza das mulheres e em domicílios chefiados pelas mesmas, na literatura há controvérsias sobre tal resultado, além do fato de não ser um fenômeno que ocorre de maneira uniforme ao longo dos anos.

Segundo Northrop (1990), em 1970 nos Estados Unidos, 50\% dos indivíduos considerados pobres estavam em domicílios chefiados por mulheres. Tal resultado pode ser explicado por dois fatores: mudança na composição demográfica da população, com o aumento da proporção de famílias chefiadas por mulheres; e aumento da diferença da ocorrência da pobreza em famílias chefiadas por mulheres em comparação às famílias com chefe masculino.

Em concordância com o trabalho supracitado, Bradshaw et al. (2003) verificaram a maior incidência da pobreza entre as mulheres no Reino Unido durante os anos de 1999 e 2000, mesmo quando algumas variáveis são controladas, como etnia, quantidade de filhos e deficiência.

Nos anos seguintes, os estudos foram expandidos e adaptados para outros países; Moghadam (1997) e Lampietti e Stalker (2000) demonstram a diversificação que há entre os países no tocante a feminização da pobreza ou sobre a representação feminina da pobreza. Nesse sentido, de acordo com Gangopadhyay e Wadhwa (2003), os domicílios indianos quando chefiados por mulheres sem cônjuge estavam mais propensos a situação de pobreza. Além disso, a incidência de pobreza aumentava quando estes domicílios estavam localizados na área urbana.

Para o caso brasileiro, Barros, Fox e Mendonça (1994) ratificaram os resultados até então verificados, após controle de diferenças regionais, concluindo que domicílios chefiados por mulheres tendem a serem mais pobres que os demais tipos de domicílios. Cabe ainda salientar que quando há crianças nesse tipo de domicílio, há elevação do grau de pobreza.

Oliveira (2018) analisou processo de feminização da pobreza em um região com elevada incidência de pobreza no Brasil: o Nordeste. A partir dos microdados da PNAD no período 2004-2015, foi estimado o Índice de Pobreza Multidimensional a fim de verificar se 
o processo de feminização da pobreza ocorre no Brasil e se sua incidência é mais elevada na região Nordeste. Os resultados apontaram que mulheres nordestinas chefes de família são multidimensionalmente mais pobres que os homens, caracterizando o processo de feminização da pobreza, diferentemente do observado para as chefes de família do país como um todo.

Indo ao encontro dos resultados já apontados, Melo (2005) concluiu que a pobreza afeta os gêneros de forma desigual, dado que as mulheres são as mais carentes quando se relaciona pobreza e gênero. A vida dupla (trabalho e lar) é um dos motivos para essa constatação, e quando elas assumem a chefia da família, na maioria dos casos, não há parceiro. Assim, nesses casos, as mulheres se tornam as únicas provedoras do sustento do lar.

Para Novellino (2004), quando o homem é o chefe, sua renda é complementada com a de sua cônjuge. Sendo assim, a tendência é que famílias chefiadas por mulheres representariam a maioria entre os pobres. Dessa forma, haveria sobrerrepresentação da pobreza feminina (OLIVEIRA, 2018).

Nesse sentido, alguns estudos como os de Moghadam (1997), Lapietti e Stalker (2000) e Costa et al. (2005) mostram a heterogeneidade dos resultados encontrados para diferentes países e que, embora ocorra feminização da pobreza, esse fenômeno não pode ser generalizado para o mundo. No momento em que são analisados outros países em desenvolvimento, Marcoux (1998) considerou dados de doze países localizados na América Latina, e constatou que é falha a suposição de que comumente os domicílios que têm mulheres em sua chefia são sobrerrepresentados entre os pobres.

De acordo com pesquisa realizada por Costa et al. (2005), foi avaliada a hipótese de que houve feminização da pobreza no Brasil ao longo do período de 1983-2003, assim como se houve sobrerrepresentação feminina da pobreza. A partir de seis definições de feminização da pobreza, foi estimado o índice de pobreza FGT que não encontrou, de modo geral, qualquer indício consistente que caracterizasse esse fenômeno. Por fim, quando foi considerada pelos autores uma distribuição intradomiciliar desigual dos recursos, persistiu o resultado de inexistência de um padrão de feminização, mas foi comprovada a sobrerrepresentação de mulheres entre os pobres.

Para Wright (1992), embora a ocorrência e intensidade da pobreza serem mais elevadas para as mulheres, não foi observado uma deterioração desse fenômeno quando observadas séries de tempo, durante os anos de 1968, 1977 e 1986 no Reino Unido. Assim, há tendência em se descartar a hipótese de feminização da pobreza. 


\section{METODOLOGIA}

Este trabalho busca entender se houve o fenômeno de feminização da pobreza no Brasil, comparando os anos 2005 e 2015. Para isso, a partir dos dados da Pesquisa Nacional por Amostra de Domicílios (PNAD), foi utilizado o indicador de pobreza FosterGreer-Thorbecke (FGT), onde três indicadores serão calculados: proporção, intensidade e severidade da pobreza.

\subsection{Indicadores de pobreza Foster-Greer-Thorbecke (FGT)}

Para a análise dos níveis de pobreza, utilizam-se os indicadores propostos por Foster, Greer e Thorbecke (1984), que demonstram vários aspectos possíveis de serem analisados sobre o fenômeno da pobreza, sendo apresentados na Equação (1).

$$
\varphi(\alpha)=\frac{1}{n z^{a}} \sum_{i=1}^{q}\left(z-\bar{y}_{1}\right)^{a}
$$

Onde:

q representa o número de famílias abaixo da linha de pobreza;

n é o tamanho da população estudada;

z é a linha de pobreza;

$\bar{y}_{1}$ é a renda per capita da i-ésima família em ordem crescente.

Assim, o índice de pobreza $F G T$ tem sua derivação com base na variação do parâmetro $\alpha$, podendo ser igual a 0,1 e 2 (HOFFMANN, 1998); sendo assim:

- $\alpha=0$ mede-se a proporção dos pobres $P_{0}$, em relação à população mundial;

- $\alpha=1$, mede-se o hiato da pobreza $P_{1}$, onde é calculada a intensidade da pobreza como déficit de renda;

- $\alpha=2$, mede-se a severidade da pobreza $P_{2}$, onde demonstra-se a desigualdade de distribuição de renda quando se analisa os mais pobres dentro da amostra.

Segundo Ravallion e Bidani (1994), o índice de intensidade da pobreza (P1) também pode ser interpretado como um potencial indicador para eliminar a pobreza através de transferências para os pobres, que são o objeto de políticas públicas. Já o indicador de severidade da pobreza é também denominado de hiato quadrático, pois mede o quadrado do hiato da pobreza em relação à linha de pobreza considerada, e dessa forma, também considera a desigualdade entre os pobres (WORLD BANK, 2009). 
Para Hagenaars (1986), os indicadores FGT têm em comum particularidades na forma axiomática que são importantes em medidas de pobreza:

I. Monotonicidade: Em uma população analisada com uma distribuição de renda característica, quando há redução de renda da família, a medida da pobreza é elevada;

II. Transferência de renda: Em uma população analisada com distribuição de renda característica, quando há a transferência de renda de uma família pobre para outra mais pobre, há aumento da medida de pobreza, visto que o nível de concentração de renda é influenciado e a compreensão de pobreza relativa é aplicado, posto que a primeira família ficou mais pobre;

III. Sensibilidade de transferências: Em uma população analisada com uma distribuição de renda característica, uma transferência de renda positiva para uma família com renda $Y$ e para uma família que possui renda $Y+d$, o impacto será positivo em maior escala para a família que possuía $Y$, devido ao seu déficit de renda.

De acordo com Lima (2008), os indicadores relativos ao hiato da pobreza e severidade da pobreza são os de maior relevância quando comparados ao de proporção de pobres, visto que aqueles levam em consideração os mais pobres dentre os pobres. Assim, atribui-se um peso maior, dada a incidência da pobreza.

As medidas de pobreza que são estabelecidas levam em consideração as informações necessárias para a formulação das linhas de indigência, e as informações dos coeficientes que representam as necessidades da residência, tais como saúde e bem-estar, para a formulação de tais linhas de pobreza. Contudo, devido a divergência e/ou ausência de informações, as linhas de pobreza adotadas são, de certo modo, divergentes, haja vista que se referem as informações de rendimento que estão em conformidade com a elevada monetarização das relações econômicas realizadas (HOFFMANN, 1998; PEREIRA, LIMA e JUSTO, 2015).

Para tanto, foi considerado o rendimento domiciliar per capita e as linhas de pobreza estabelecidas de $1 / 2$ salário mínimo para os anos de 2005 e 2015, correspondentes a $R \$$ 150,00 e $R \$ 394,00$, respectivamente; e de 1/4 de salário mínimo (extrema pobreza) de $\mathrm{R} \$ 75,00$ e $\mathrm{R} \$ 197,00^{1}$ para os anos de 2005 e 2015 . Tais valores foram utilizados, levando-

\footnotetext{
${ }^{1}$ Ou seja, em situação de pobreza considera-se as linhas de pobreza oficiais adotadas pelo governo. As linhas de pobreza estabelecidas definem as famílias consideradas pobres (ou em situação de vulnerabilidade) e em situação de extrema pobreza.
} 
se em consideração o Decreto $N^{\circ}$ 6.135, de 26 de junho 2007, e a Lei $n^{\circ} 8.742$, de 7 de dezembro de 1993, como referências, que pormenorizam as famílias que estão em situações de instabilidade e extrema pobreza.

\subsubsection{Dados}

A base de dados utilizada para este estudo é a Pesquisa Nacional por Amostra de Domicílios (PNAD), presente no Instituto Brasileiro de Geografia e Estatística (IBGE), para os anos de 2005 e 2015. A PNAD aborda anualmente, de maneira geral, características populacionais, educacionais, rendimento e habitação, migração, fecundidade, entre outros (IBGE, 2018). Dado que 2015 é o ano com a base de dados mais recente disponibilizada pelo IBGE de forma não contínua, buscou-se avaliar se houve o fenômeno de feminização da pobreza no Brasil em uma janela de referência de dez anos. Assim, o ano inicial de análise será o ano de 2005.

\section{RESULTADOS}

Nesta seção, são apresentados os principais resultados obtidos na pesquisa. Inicialmente, é realizada análise geral dos indicadores de pobreza para o Brasil e suas macrorregiões e, posteriormente será verificado se houve feminização da pobreza no Brasil nos anos 2005 e 2015. O fenômeno de feminização da pobreza ocorrerá quando houver crescimento (absoluto ou relativo) ${ }^{2}$ para as mulheres. Em termos absolutos, quando houver crescimento da pobreza para as mulheres, e termos relativos, se o grupo das mulheres apresentar redução da pobreza em menor grau em relação à redução para o grupo dos homens - considerando o mesmo para mulheres chefes, mulheres cônjuges e famílias chefiadas por mulheres (família monoparental).

Sendo assim, são apresentados os indicadores de pobreza e extrema pobreza de Foster-Greer-Thorbecke (FGT) para o Brasil e regiões, dividindo-se a distribuição por gênero, tipo de família e posição na ocupação, considerando igualdade intrafamiliar dos rendimentos.

${ }^{2} \mathrm{~A}$ definição de feminização da pobreza é baseada em Costa et al. (2005). 


\subsection{Pobreza no Brasil e suas macrorregiões}

A partir das duas linhas de pobreza estimadas (pobreza e de extrema pobreza), são apresentadas na Tabela 1 as estimativas do perfil da pobreza brasileira, obtida pelo método de Foster-Greer-Thorbecke (1984). O índice P0 mede a proporção de pessoas que não possuem renda per capita superior à linha de pobreza (R\$150,00 para 2005 e $R \$ 394,00$ para 2015). Além deste, são calculados o P1 (hiato da pobreza) e o P2 (severidade da pobreza), o que permite mais informações relevantes a respeito dos mais pobres.

Tabela 1 - Indicadores de pobreza FGT (Foster-Greer-Thorbecke) para renda per capita inferior a meio salário mínimo e a um quarto de salário mínimo para o Brasil e macroregiões - 2005 e 2015

\begin{tabular}{|c|c|c|c|c|c|c|c|c|}
\hline \multicolumn{2}{|l|}{ Ano } & Indicador & Brasil & Norte & Nordeste & Sudeste & Sul & $\begin{array}{l}\text { Centro- } \\
\text { Oeste }\end{array}$ \\
\hline \multirow{6}{*}{2005} & \multirow{3}{*}{ Pobreza } & P0 & 0.2720 & 0.4420 & 0.5313 & 0.1540 & 0.1580 & 0.2176 \\
\hline & & P1 & 0.1124 & 0.1857 & 0.2529 & 0.0529 & 0.0563 & 0.0781 \\
\hline & & P2 & 0.0614 & 0.1003 & 0.1511 & 0.0252 & 0.0280 & 0.0379 \\
\hline & \multirow{3}{*}{$\begin{array}{l}\text { Pobreza } \\
\text { Extrema }\end{array}$} & P0 & 0.0912 & 0.1568 & 0.2365 & 0.0329 & 0.0381 & 0.0485 \\
\hline & & P1 & 0337 & 00514 & 0.0951 & 0.0107 & 0.0132 & 0.0163 \\
\hline & & $\mathrm{P} 2$ & 0.0177 & 0.0252 & 0.0523 & 0.0052 & 0.0070 & 0.0083 \\
\hline \multirow{6}{*}{2015} & \multirow{3}{*}{ Pobreza } & P0 & 0.2051 & 0.3652 & 0.3976 & 0.1229 & 0.0982 & 0.1277 \\
\hline & & P1 & 0.0788 & 0.1465 & 0.1766 & 0.0384 & 0.0316 & 0.0389 \\
\hline & & P2 & 0.0412 & 0.0777 & 0.0777 & 0.0171 & 0.0148 & 0.0168 \\
\hline & \multirow{3}{*}{$\begin{array}{l}\text { Pobreza } \\
\text { Extrema }\end{array}$} & P0 & 0.0596 & 0.1171 & 0.1577 & 0.0202 & 0.0209 & 0.0186 \\
\hline & & P1 & 0.0213 & 0.0401 & 0.0609 & 0.0061 & 0.0062 & 0.0058 \\
\hline & & P2 & 0.0110 & 0.0198 & 0.0327 & 0.0029 & 0.0028 & 0.0029 \\
\hline
\end{tabular}

Fonte: Elaborado pelos autores com base nos microdados das PNADs 2005 e 2015.

No Brasil, para o ano de 2005, a partir do cálculo do índice $F G T$, considerando toda a população economicamente ativa, a proporção de pobres (P0) é de aproximadamente $27,20 \%$. Por sua vez, o hiato da pobreza (P1) e a severidade da pobreza (P2) foram estimados em $11,24 \%$ e $6,14 \%$, respectivamente. Já para o ano de 2015 , é possível observar que há queda nos os indicadores de pobreza. No ano supracitado, os valores são de $20,51 \%, 7,88 \%$ e 4,12\% para P0, P1 e P2, respectivamente. A redução nos indicadores ocorre também para a linha de pobreza referente a um quarto de salário mínimo, que considera os brasileiros em situação de pobreza extrema. 
Quanto às macrorregiões, Nordeste e Norte são aquelas que apresentam indicadores de pobreza mais elevados. Em 2005, no Sudeste, Sul e Centro-Oeste, a proporção de pobres foi estimada em 15,40\%, 15,80\% e 21,76\%, apresentando redução em 2015 , onde atingiram as cifras de $12,30 \%, 9,82 \%$ e $12,77 \%$, respectivamente. Por sua vez, as regiões Nordeste e Norte apresentam sempre os maiores valores para os indicadores P0, P1 e P2. Em um estudo realizado por Pereira (2017), o mesmo resultado é encontrado, e o autor chama a atenção para a discussão em termos de disparidade em termos de condições de vida das pessoas que residem em regiões cujas atividades ligadas ao meio rural predominam (caso das regiões citadas) em relação àquelas que habitam regiões com dinâmicas produtivas mais intensas.

Especificamente, a maior proporção de pobres é observada para a região Nordeste $^{3}$, atingindo aproximadamente 53,13\% em 2005, e apesar de apresentar redução significativa em 2015, ainda é aquela com a maior proporção de pobres, quase 40\%. Em seguida, vem a região Norte com proporção de pobres de 44,20\% em 2005 e aproximadamente 36,52\% em 2015. O grau de inferioridade na média de rendimento se mostra bastante intenso nas duas regiões, com P1 estimado em 2015 de 17,66\% e 14,65\%, respectivamente. O P2, que representa a desigualdade entre a população pobre, também é relativamente elevado nessas duas regiões em ambos os anos analisados, atingindo $16 \%$ no Nordeste e $10 \%$ no Norte, em 2005.

No tocante a extrema pobreza, as duas regiões ainda se destacam pelos indicadores elevados. Entre 2005 e 2015, a proporção de pobres apresentou queda considerável, mas ainda assim é elevada. A região Norte apresentou decrescimento de 15,68\% para 11,71\% em 2015 e no Nordeste a queda foi da ordem 23,65\% para 15,77\% no período.

Comparando-se a linha de pobreza de apenas $1 / 2$ salário mínimo apresentada na Tabela anterior, é possível observar que a proporção de pessoas vivendo em situação de extrema pobreza, com $1 / 4$ de salário mínimo, nas regiões Norte e Nordeste, é superior ao número de pessoas pobres das demais regiões. Tais resultados denotam a grande desigualdade distributiva de renda no Brasil, uma vez que a maior parte da população em condições de pobreza e extrema pobreza concentra-se nas duas regiões.

\footnotetext{
${ }^{3} \mathrm{~A}$ maior parte da população brasileira de baixa renda se encontra no Norte e Nordeste. Os maiores rendimentos estão concentrados em municípios do Sudeste e do Sul do Brasil. Já no Centro-Oeste, as rendas são relativamente elevadas (OXFAM BRASIL, 2017).
} 


\subsection{Indicadores de pobreza para as mulheres}

A seguir são apresentados os indicadores de incidência (P0), intensidade (P1) e severidade (P2) da pobreza para homens e mulheres nos anos analisados. A hipótese de que há feminização da pobreza será confirmada em dois casos: i) absoluto, se no período houver aumento nos indicadores de pobreza para as mulheres; e ii) relativo, se a situação das mulheres piorar comparativamente à dos homens. Dessa forma, se ambos apresentarem redução nos indicadores de pobreza, mas os homens apresentarem redução mais elevada, então a hipótese de feminização da pobreza será confirmada (COSTA et al., 2005).

Para cada uma das linhas de pobreza foi calculada a incidência, intensidade e severidade da pobreza para mulheres e homens. As Figuras 1, 2 e 3 apresentam a proporção, intensidade e severidade da pobreza, considerando-se $1 / 2$ salário mínimo para a linha de pobreza e 1/4 de salário mínimo para a linha de extrema pobreza.

Entre 2005 e 2015, é possível observar uma tendência de queda nos valores dos indicadores P0, P1 e P2 para as mulheres nas duas linhas de pobrezas levadas em consideração. Para os homens, a proporção de pobres se manteve constante; e a intensidade apresentou pequeno aumento, assim como a severidade da pobreza.

Figura 1- Evolução da incidência da pobreza para mulheres e homens

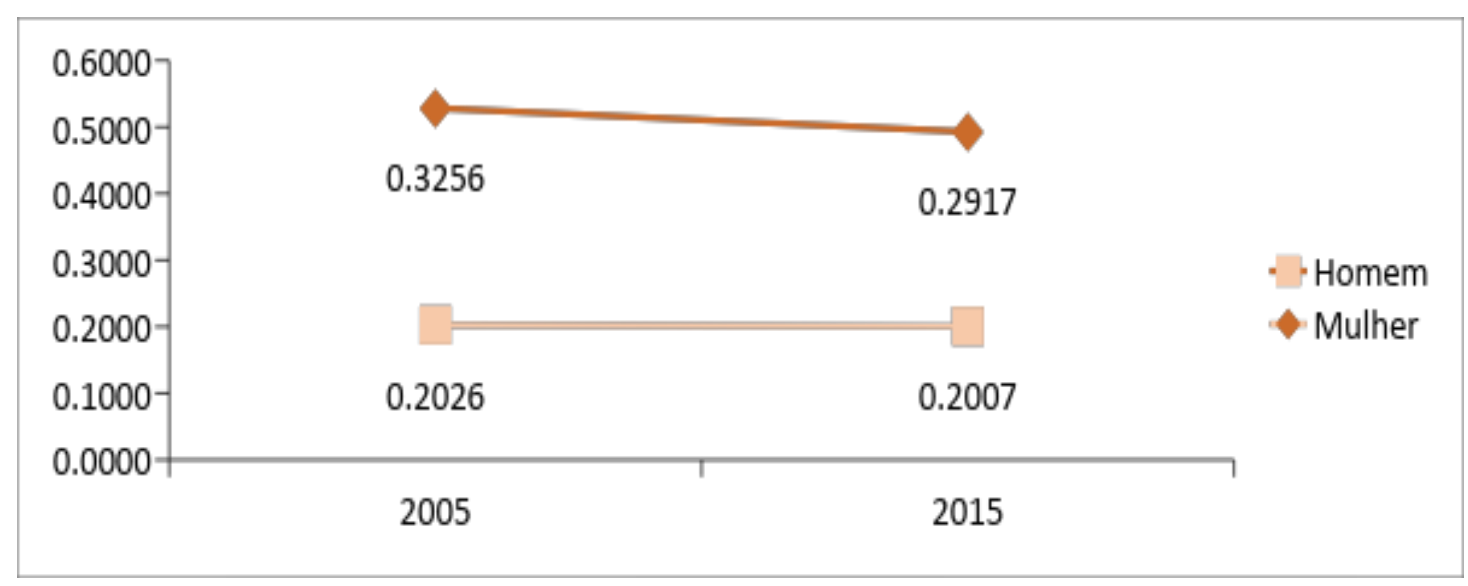

Fonte: Elaborado pelos autores com base nos microdados das PNADs 2005 e 2015. 
Figura 2 - Evolução da intensidade da pobreza para mulheres e homens

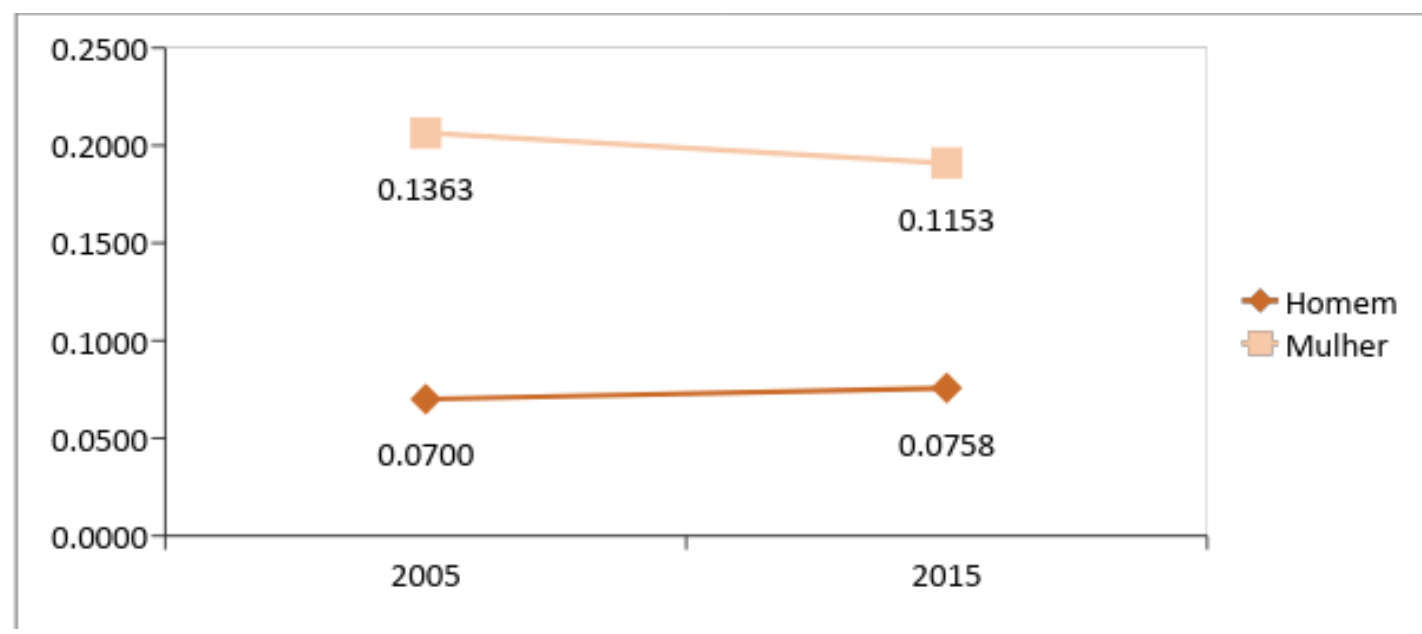

Fonte: Elaborado pelos autores com base nos microdados das PNADs 2005 e 2015.

Figura 3 - Evolução da severidade da pobreza para mulheres e homens

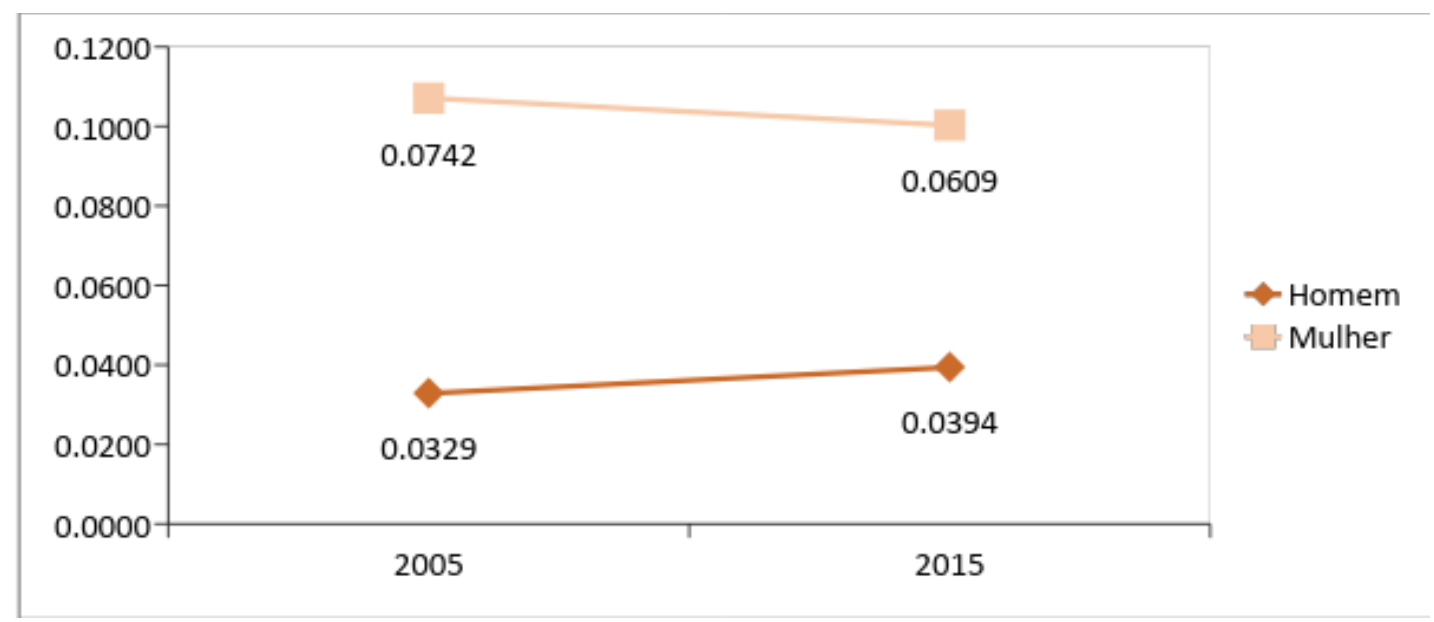

Fonte: Elaborado pelos autores com base nos microdados das PNADs 2005 e 2015.

Observando-se a Figura 4 para a linha de extrema pobreza, considerando $1 / 4$ do salário mínimo, os indicadores para os homens apresentaram aumento quanto a incidência da extrema pobreza, que passou de $4,13 \%$ para $5,71 \%$. No caso das mulheres, houve redução de $10,08 \%$ para $8,84 \%$ no período analisado.

Através da Figura 5 é possível observar que a intensidade da extrema pobreza se manteve constante para as mulheres, em torno de $3 \%$. Ainda assim esta foi superior àquela verificada para os homens, que apresentou elevação de 1,32\% para 2,02\% entre 2005 e 2015. 
Quanto ao indicador de severidade da pobreza, as mulheres obtiveram pequena redução. Em contrapartida, no caso dos homens houve aumento de 1,31\% para 2,02\%, como demonstrado na Figura 6.

Figura 4 - Evolução da incidência da extrema pobreza para mulheres e homens.

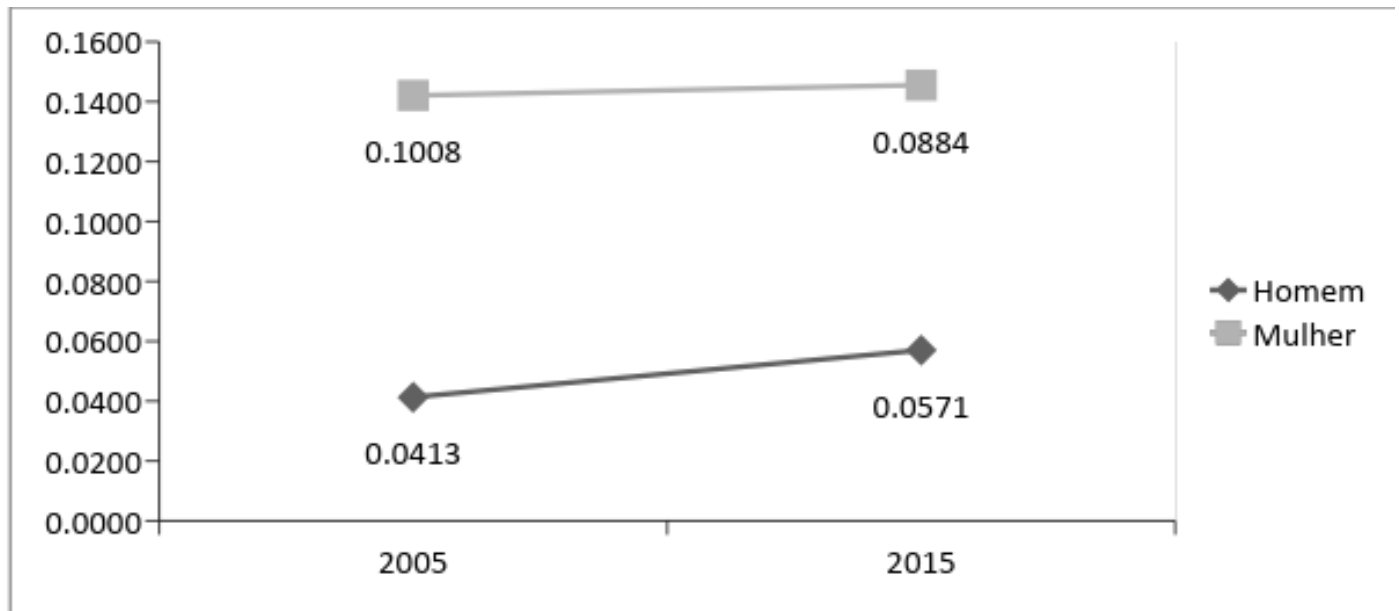

Fonte: Elaborado pelos autores com base nos microdados das PNADs 2005 e 2015.

Figura 5 - Evolução da intensidade da extrema pobreza para mulheres e homens

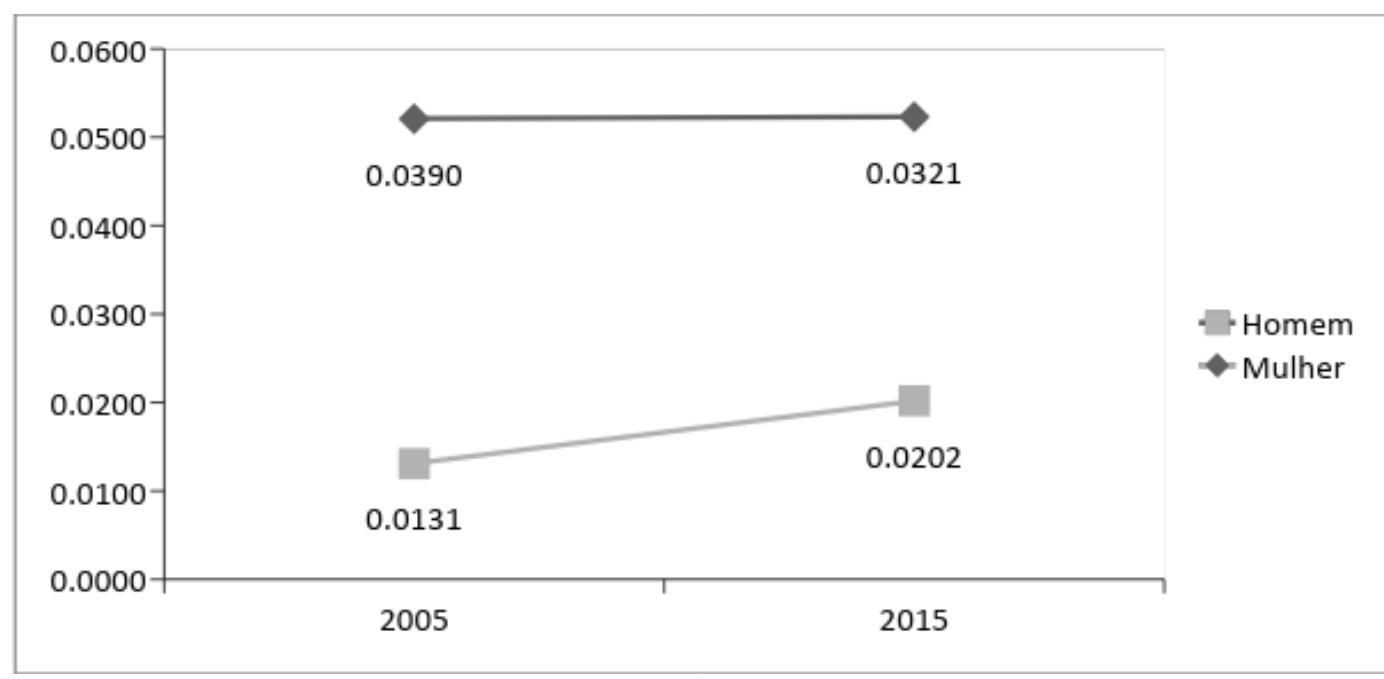

Fonte: Elaborado pelos autores com base nos microdados das PNADs 2005 e 2015. 
Figura 6 - Evolução da severidade da extrema pobreza para mulheres e homens

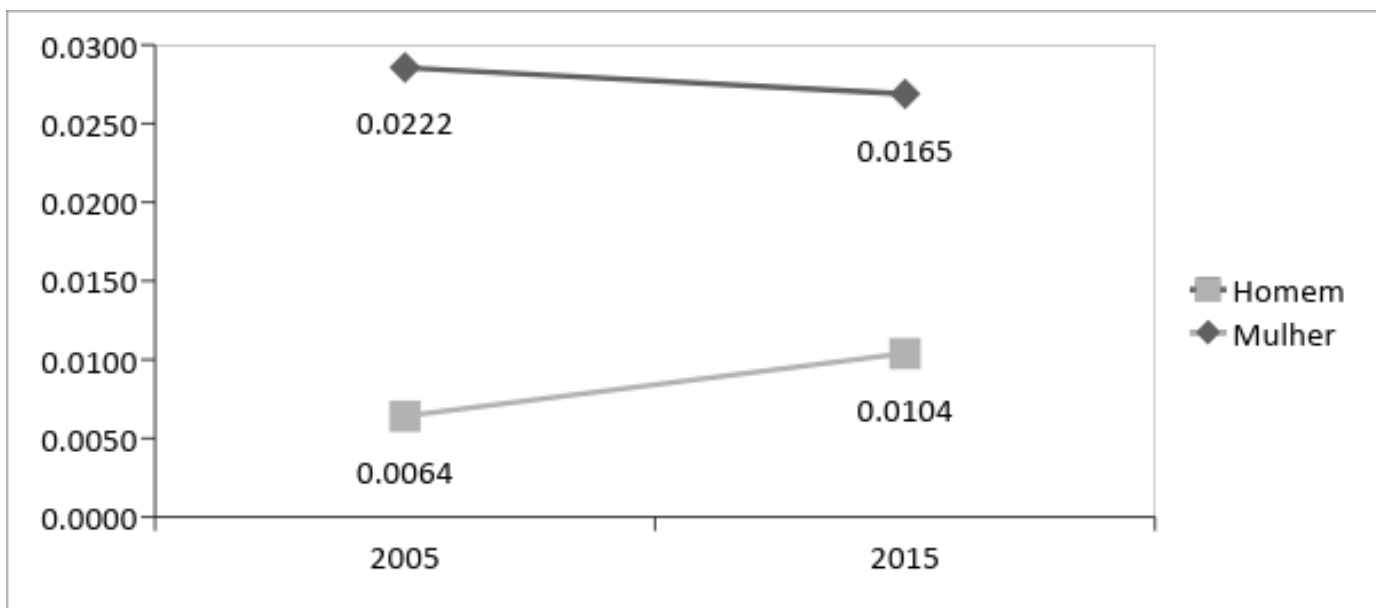

Fonte: Elaborado pelos autores com base nos microdados das PNADs 2005 e 2015.

Analisando-se os diferenciais em relação aos indicadores de pobreza P0, P1 e P2 entre 2005 e 2015, é possível observar tendência de redução para todos eles entre as mulheres, indicando que não há a confirmação da hipótese de feminização da pobreza, segundo sua definição absoluta. Os indicadores para os dois gêneros não se comportaram de forma idêntica, mas as mulheres apresentaram resultados relativamente positivos. Sendo assim, pode-se concluir que também não ocorreu aumento relativo da pobreza entre as mulheres. Ou seja, não houve qualquer processo de feminização da pobreza no período analisado - absoluto ou relativo. Tais resultados vão ao encontro dos encontrados por Lavinas (1996) e Costa et al. (2005).

\subsection{Evolução dos indicadores de pobreza, segundo a posição na ocupação}

Esta subseção considerará a mesma análise realizada anteriormente, mas agora levando em consideração somente os membros chefes ou cônjuges, que a priori são os que mais colaboram com o rendimento familiar. $O$ objetivo é capturar diferenças entre homens e mulheres no que tange o acometimento pela pobreza.

A feminização da pobreza de 2005 para 2015 será confirmada em dois casos: i) absoluto, se no período houver aumento nos indicadores de pobreza para "mulher chefe" e em uma segunda análise para "mulher cônjuge"; e ii) relativo, se as situações da "mulher chefe" ou "mulher cônjuge" piorarem comparativamente com "homem chefe" ou "homem cônjuge". Dessa forma, se ambos apresentarem redução nos indicadores de pobreza, mas 
os homens na condição de chefes e cônjuges apresentarem redução mais elevada, então a hipótese de feminização será confirmada (COSTA et al., 2005).

A Tabela 2 mostra a evolução dos indicadores de pobreza, P0, P1 e P2 para os diferentes tipos de famílias, segundo as duas linhas de pobreza: 1/2 salário mínimo e de 1/4 de salário mínimo nos anos analisados.

Inicialmente, considerando-se a linha de pobreza ( $1 / 2$ salário mínimo), pode-se observar que a incidência da pobreza (P0), quando consideradas apenas "mulheres chefes", é mais elevada nos dois anos analisados em aproximadamente 35,50\%. Entretanto, esta não foi a categoria que apresentou maior elevação. Pelo contrário, a proporção de pobres se manteve quase inalterada. Nesse caso, cabe ressaltar que na condição de cônjuge, as mulheres em 2005 apresentaram quase o mesmo percentual em relação aos homens quando chefes. Em contrapartida, houve aumento substancial para a "mulher cônjuge".

Tabela 2 - Indicadores de pobreza FGT (Foster-Greer-Thorbecke) para renda per capita inferior a meio salário e a um quarto de salário, segundo a posição na ocupação para os anos de 2005 e 2015

\begin{tabular}{|c|c|c|c|c|c|c|}
\hline Ano & & Indicador & $\begin{array}{c}\text { Mulher } \\
\text { Chefe }\end{array}$ & $\begin{array}{c}\text { Homem } \\
\text { Chefe }\end{array}$ & $\begin{array}{l}\text { Mulher } \\
\text { cônjuge }\end{array}$ & $\begin{array}{l}\text { Homem } \\
\text { cônjuge }\end{array}$ \\
\hline \multirow{6}{*}{2005} & \multirow{3}{*}{ Pobreza } & P0 & 0.3514 & 0.1757 & 0.1716 & 0.2200 \\
\hline & & P1 & 0.1262 & 0.0630 & 0.0575 & 0.0791 \\
\hline & & P2 & 0.0590 & 0.0313 & 0.0259 & 0.0385 \\
\hline & \multirow{3}{*}{$\begin{array}{l}\text { Pobreza } \\
\text { Extrema }\end{array}$} & P0 & 0.0699 & 0.0419 & 0.0348 & 0.0493 \\
\hline & & P1 & 0.0220 & 0.0144 & 0.0084 & 0.0167 \\
\hline & & P2 & 0.0107 & 0.0076 & 0.0029 & 0.0086 \\
\hline \multirow{6}{*}{2015} & \multirow{3}{*}{ Pobreza } & PO & 0.3589 & 0.1823 & 0.2176 & 0.2147 \\
\hline & & $\mathrm{P} 1$ & 0.1356 & 0.0698 & 0.0796 & 0.0823 \\
\hline & & P2 & 0.0687 & 0.0369 & 0.0404 & 0.0432 \\
\hline & \multirow{3}{*}{$\begin{array}{l}\text { Pobreza } \\
\text { Extrema }\end{array}$} & P0 & 0.0956 & 0.0544 & 0.0575 & 0.0628 \\
\hline & & $\mathrm{P} 1$ & 0.0324 & 0.0198 & 0.0193 & 0.0225 \\
\hline & & P2 & 0.0164 & 0.0102 & 0.0097 & 0.0116 \\
\hline
\end{tabular}

Fonte: Elaborado pelos autores com base nos microdados das PNADs 2005 e 2015. 
A análise dos resultados revela que a intensidade da pobreza (P1) é semelhante ao perfil de incidência da pobreza, de acordo com a posição na ocupação. O índice de intensidade, quando se utiliza a linha de pobreza correspondente a 1/2 salário mínimo, é maior na condição de "mulher chefe" em ambos os anos. Já em relação ao "homem chefe", a mulher na condição de cônjuge, em 2015, apresentou índice mais elevado, alcançando $7,96 \%$.

Para a linha de extrema pobreza, quanto a incidência da pobreza (P0), as mulheres chefes de família apresentaram resultado semelhante à linha de pobreza de $1 / 2$ salário mínimo, onde são a maioria entre os pobres. Entretanto, o crescimento da incidência da pobreza para as mulheres foi mais significativo em 2015, 2,58 pontos; em relação aos homens chefes de família, "mulher cônjuge" e "homem cônjuge", com valores de 1,26 p.p, 2,27 p.p e 1,35 p.p, respectivamente. Mais uma vez, quando a categoria em que a mulher está inserida não apresenta os maiores percentuais, as elevações são mais substanciais em relação a posição ocupada pelos homens. Para o hiato da pobreza (P1) e severidade da pobreza (P2), houve crescimento para todas as posições na ocupação.

Assim, de acordo com os resultados apresentados, pode-se constatar que o comportamento difere ao encontrado anteriormente para homens e mulheres. Entre 2005 e 2015, tanto na incidência quanto na intensidade e na severidade da pobreza, houve aumento para ambos os gêneros. Entretanto, quando é observada a variável mulher chefe de família, é possível observar os maiores níveis dos indicadores.

Homens na mesma posição, em 2005 e 2015, compreendiam a proporção de 17\% a $18 \%$ entre os pobres. Em contrapartida, as mulheres permanecem com taxa superior, aproximadamente $35 \%$ em ambos os anos. O mesmo ocorre quanto à pobreza extrema, onde elas apresentam uma maior proporção, em média, 3,6 pontos percentuais a mais que os homens. Os demais indicadores, incidência e severidade da pobreza, nas duas linhas de pobreza consideradas, apresentam valores mais elevados para as brasileiras. Ou seja, as mulheres possuem piores condições quando comparadas aos homens, na mesma posição de ocupação nos domicílios.

A ausência do cônjuge ou companheiro no ambiente familiar indica que as mulheres podem estar em situação de maior vulnerabilidade social, pois não possuem em suas residências uma rede primária de proteção e geração de renda que é a família. Essa informação também sugere que a mulher pode ter sido reconhecida como chefe pela ausência de um homem na família, realidade que contribui e acentua a vulnerabilidade à 
pobreza por parte da mulher. Isso porque na condição de exclusiva mantenedora do lar, ao tornarem-se chefes de família, essa vulnerabilidade se agrava (OLIVEIRA, 2018).

Em síntese, em termos absolutos, pode-se constatar que houve o fenômeno da feminização da pobreza para o Brasil nos anos de 2005 para 2015, considerando-se a posição na ocupação das mulheres (se chefe ou se cônjuge). Resultado semelhante foi verificado por Barros, Fox e Mendonça (1994). Em termos relativos, como nenhum indicador apresentou redução da pobreza, conclui-se que não houve processo de feminização da pobreza no Brasil. Castro (1989) afirma que os domicílios chefiados tanto por homens ou mulheres sofrem de maneira semelhante com os baixos níveis de renda.

\subsection{Evolução dos indicadores de pobreza segundo o tipo de família}

Nesta subseção, são apresentados os indicadores de incidência (P0), intensidade (P1) e severidade (P2) da pobreza de acordo com o tipo de família. A feminização da pobreza entre 2005 e 2015 será confirmada, assim como nas seções anteriores, em dois casos: i) absoluto, se no período houver aumento nos indicadores de pobreza para as famílias do tipo "mulher com filhos"; e ii) relativo, se a situação da "mulher com filhos" piorar em relação ao "casal com filhos" (COSTA, et al., 2005). Dessa forma, se ambos apresentarem redução nos indicadores de pobreza, mas "casal com filhos" apresentar redução mais elevada a observada para a "mulher com filhos", então a hipótese de feminização será confirmada. Os outros tipos de família estão inseridos na análise para representar os outros tipos de família e enriquecer a discussão.

A estimação dos indicadores de pobreza para o tipo de família é apresentada na Tabela 3. Os resultados apresentados para os índices FGT mostram que quando se tem a presença de filhos na família, estes se mostram superiores em comparação aos demais tipos de famílias. A proporção de famílias do tipo "mulher com filhos", cuja renda é inferior a $1 / 2$ salário mínimo (P0), em 2005, é de 38,89\%, com aumento em 2015 para 39,63\%. Já para o tipo de família "casal com filhos", essa razão permanece constante.

Em relação ao hiato da pobreza $(P 1)$, os índices são de 15,47\% e 11,60\%, respectivamente, e a severidade da pobreza (P2), que se manteve constante para famílias do tipo "casal com filhos" e "mulher com filhos", apresentou aumento de menos de 1 ponto percentual. Tendo em vista a significância estatística do teste de igualdade entre os índices, pode-se constatar que a presença de filhos na família tende a aumentar a pobreza destas, 
como apresentado nos resultados. Ainda assim, considerando a presença de filhos, as mulheres sem cônjuge estão em situação mais vulnerável comparativamente àquelas em ambiente com a presença destes.

Considerando a linha de extrema pobreza para 2005, pode-se observar que a incidência da pobreza para todos os tipos de família foi menor em relação a 2015. É interessante ressaltar que mesmo a família na condição de "mulher com filhos" representando a maior proporção de pobres, o tipo de família que apresentou maior aumento (2,19 pontos percentuais), de 2005 para 2015, foi a condição "casal com filhos".

Tabela 3- Indicadores de pobreza FGT (Foster-Greer-Thorbecke) para renda per capita inferior a meio salário mínimo e um quarto do salário mínimo, segundo o tipo de família 2005 e 2015.

\begin{tabular}{|c|c|c|c|c|c|c|}
\hline \multicolumn{2}{|l|}{ Ano } & Indicador & $\begin{array}{c}\text { Casal } \\
\text { sem } \\
\text { filhos }\end{array}$ & $\begin{array}{l}\text { Casal } \\
\text { com } \\
\text { filhos }\end{array}$ & $\begin{array}{c}\text { Mulher } \\
\text { com } \\
\text { filhos }\end{array}$ & $\begin{array}{c}\text { Outro } \\
\text { tipo }\end{array}$ \\
\hline \multirow{6}{*}{2005} & \multirow{3}{*}{ Pobreza } & P0 & 0.06650 & 0.30680 & 0.38890 & 0.18970 \\
\hline & & P1 & 0.02470 & 0.10920 & 0.14830 & 0.06740 \\
\hline & & P2 & 0.01300 & 0.05180 & 0.07620 & 0.03280 \\
\hline & \multirow{3}{*}{$\begin{array}{l}\text { Pobreza } \\
\text { Extrema }\end{array}$} & P0 & 0.01470 & 0.06190 & 0.10830 & 0.04490 \\
\hline & & $\mathrm{P} 1$ & 0.00710 & 0.02030 & 0.03790 & 0.01430 \\
\hline & & $\mathrm{P} 2$ & 0.00500 & 0.01030 & 0.01830 & 0.00700 \\
\hline \multirow{6}{*}{2015} & \multirow{3}{*}{ Pobreza } & P0 & 0.08700 & 0.30790 & 0.39630 & 0.20890 \\
\hline & & $\mathrm{P} 1$ & 0.03500 & 0.11600 & 0.15470 & 0.07930 \\
\hline & & P2 & 0.01970 & 0.05960 & 0.08120 & 0.04130 \\
\hline & \multirow{3}{*}{$\begin{array}{l}\text { Pobreza } \\
\text { Extrema }\end{array}$} & P0 & 0.02820 & 0.08380 & 0.12370 & 0.06090 \\
\hline & & $\mathrm{P} 1$ & 0.01180 & 0.02960 & 0.04200 & 0.02120 \\
\hline & & P2 & 0.00680 & 0.01520 & 0.02040 & 0.01060 \\
\hline
\end{tabular}

Fonte: Elaborado pelos autores com base nos microdados das PNADs 2005 e 2015.

Quanto ao gap de pobreza ou intensidade da pobreza (P1), os tipos de família "casal com filhos" e "mulher com filhos" apresentam índices de intensidade mais elevados, visto que "mulher com filhos" apresentou o maior valor entre todas as demais. Já a família do tipo "casal sem filhos", da mesma forma que ocorreu com o perfil de incidência, apresentou 
os menores índices entre todos os tipos de família. Ou seja, "casal sem filhos" estão mais próximos da linha de pobreza.

Assim, é possível constatar que o comportamento tanto da incidência quanto da intensidade e severidade da pobreza no período analisado para cada tipo de família não é homogêneo. Outro resultado notável é o fato de que as famílias do tipo "casal com filhos" e "mulher com filhos" apresentam sempre os indicadores de pobreza mais elevados.

De acordo com os resultados, em termos absolutos, pode-se concluir que houve o fenômeno da feminização da pobreza para o Brasil entre os anos de 2005 e 2015, considerando o tipo de família "mulher com filhos. Em média, a variável "mulher com filhos" apresentou proporção entre os pobres superior à variável "casal com filhos" em cerca de 8,5 pontos percentuais. Em termos de extrema pobreza, "mulheres com filhos" em 2005 representavam 10,83\% entre os pobres, passando em 2015 para 12,37\%. Já o "casal com filhos" apresentou proporção de pobreza de 6,19\% e 8,38\% nos anos analisados, respectivamente.

Em termos relativos, como nenhum indicador apresentou redução da pobreza, conclui-se que não houve processo de feminização da pobreza no Brasil. O mesmo resultado foi encontrado por Costa et al.(2005). No estudo dos referidos autores, após verificar a hipótese de que houve o processo de feminização da pobreza entre 1983 e 2003, os resultados indicaram que não houve qualquer padrão de feminização da pobreza no Brasil, considerando indicadores de pobreza para mulheres e para pessoas pertencentes a famílias chefiadas por mulheres sem companheiros.

\section{CONSIDERAÇÕES FINAIS}

O objetivo do presente estudo foi verificar a existência de "feminização da pobreza" no Brasil nos anos de 2005 e 2015. Tal temática tem sido discutida na literatura com o intuito de verificar a situação da mulher em relação a incidência da pobreza, o que evidencia mais um dos possíveis efeitos de desigualdades em termos de gênero.

Na literatura, não é consenso que as mulheres ou famílias chefiadas por elas estão empobrecendo mais. Sendo assim, em termos de análise, considerou-se que a hipótese de feminização da pobreza seria confirmada caso para o grupo das mulheres, a pobreza apresentasse crescimento (absoluto ou relativo). Em termos absolutos, havendo crescimento da pobreza para as mulheres, e termos relativos, se o grupo das mulheres 
apresentasse redução da pobreza em menor grau em relação à redução para o grupo dos homens - considerando o mesmo para mulheres chefes, mulheres cônjuges e famílias chefiadas por mulheres.

A partir dos dados das PNAD's 2005 e 2015 e dos indicadores de pobreza FosterGreer-Thorbecke (FGT) foi calculado a proporção de pobres (P0) em relação ao restante da população, o hiato (P1) e a severidade (P2) da pobreza. Foram utilizadas duas linhas de pobreza na análise, uma levando em consideração $1 / 2$ salário mínimo e outra de $1 / 4$ de salário mínimo para 2005 e 2015, respectivamente.

Os principais resultados indicaram que quando se considera apenas homens e mulheres, não há qualquer evidência de feminização da pobreza para o Brasil no período analisado. Em outro ponto da análise, observando-se a evolução dos indicadores de pobreza, segundo a posição na ocupação da mulher (se chefe ou se cônjuge) e o tipo de família (mulher com filhos), concluiu-se que em termos absolutos ocorreu o processo de feminização da pobreza, uma vez que os indicadores de incidência e intensidade aumentaram de 2005 para 2015. Em termos relativos, não houve redução dos indicadores, e portanto, não houve confirmação da hipótese de feminização da pobreza.

$\mathrm{Na}$ literatura, ainda são escassos trabalhos que associem pobreza e gênero, dois temas que compõem uns dos mais graves problemas enfrentados pelo Brasil e outros países em desenvolvimento. Sendo assim, o presente estudo contribui para a literatura. Além disso, os resultados aqui apresentados podem incentivar a implementação de futuras políticas públicas de combate à pobreza e desigualdade de gênero.

\section{REFERÊNCIAS}

ABRAMO, L. Desigualdade de gênero e raça no mercado de trabalho brasileiro. Ciência e Cultura. vol.58 n.4 São Paulo outubro/dezembro. 2006.

BANCO MUNDIAL. 2013. Poverty and Equity Data. Disponível em<http://povertydata.worldbank. org/poverty/home>. Acesso em 23 dez 2018.

BARRETO, F. A. F. D.; OLIVEIRA, V. H. DE; FRANÇA, J. M. de. O que mais importa no combate à pobreza, crescimento econômico ou redução da desigualdade: evidências para as regiões brasileiras.Fortaleza: LEPCAEN (SérieEnsaiossobrePobreza, n. 16), 2018.

BARROS, R. P.; FOX, L.; MENDONÇA, R. Female-Headed households, poverty, and the welfare of children in urban

Brazil. Whashington, DC: The World Bank, Mar.1994 (Policy Research Working Paper, n. 1. 275). 
BRADSHAW, J. FINCH, N.; KEMP, P. A.; MAYHEW, E.; WILLIAMS, J. Gender and poverty in Britain. York: Social PolicyResearch Unit, University of York, (Working Paper Series, n. 6), 2013.

BUAINAIN, A. M.; ALVES, E.; SILVEIRA, J. M. da.; NAVARRO, Z. Sete teses sobre o mundo rural brasileiro. Revista de Política Agrícola, Vol. 22, №. 2, Brasília, Distrito Federal, p. 105-121, abril-junho, 2013.

CASTRO, M. G. Family, gender and work: the case of female heads of houselod in Brazil (states of São Paulo and Bahia) - 1950 - 1980. University of Florida, 1989.

COSTA, J. S; PINHEIRO, L; MEDEIROS, M; QUEIROZ, C. A face feminina da pobreza: sobre-representação e feminização da pobreza no Brasil. Brasília, DF: IPEA, 2005. (Texto para discussão, n. 1137). Disponível em: <http://www.ipea.gov.br/portal/images/stories/PDFs/TDs/td_1137.pdf>. Acesso em: 20 dez 2018.

FOSTER, J.; GREER, J.; THORBECKE, E. A Class of Decomposable Poverty Measures. Econometrica, Vol. 52, No. 3, p. 761-766, 1984.

GUIMARÃES, P. W. Variação de renda familiar, desigualdade e pobreza no Brasil. $177 f$. Tese (doutorado em Economia Aplicada) - Departamento de Economia Rural,Universidade Federal de Viçosa, Minas Gerais, 2007.

HOFFMANN, R. Distribuição de renda: medidas de desigualdade e pobreza. São Paulo: EDUSP, 1998.

INSTITUTO BRASILEIRO DE GEOGRAFIA E ESTATÍSTICA (IBGE). Microdados da Pesquisa Nacional por Amostra de Domicílios PNAD. Rio de Janeiro, RJ, 2018.

KAGEYAMA, A. O Sub-emprego agrícola nos anos 90. Texto para Discussão. Campinas, Instituto de Economia, UNICAMP, março, 1997.

KAGEYAMA, A. e HOFFMANN, R. Pobreza no Brasil: uma perspectiva multidimensional. Economia e Sociedade, Campinas, v15, n. 1 (26), p. 79-112, jan./jun. 2006.

KUZNETS, S. Economic Growth and Income Inequality. American Economic Review, v.45, n.1, 1955.

LAMPIETTI, J. A.; STALKER, L. Consumption expenditure and female poverty: a review of the evidence. Washington, DC: Policy Research Report on Gender and Development, The World Bank, Apr. 2000 (Working Paper Series, n. 11).

LAVINAS, Lena. As mulheres no universo da pobreza: o caso brasileiro. Estudos Feministas, v.4, n. 2, p. 464-479, 1996.

LIMA, J. R. F. de. Efeitos da pluriatividade e rendas não-agrícola sobre a pobreza e desigualdade rural na região Nordeste. 157 f. Tese (Doutorado em Economia Aplicada) 
- Departamento de Economia Rural, Universidade Federal de Viçosa, Viçosa, Minas Gerais, 2008.

MARCOUX, A. The feminization of poverty: claims, facts, and data needs. PopulationandDevelopmentReview, v. 24, n. 1, p. 131-139, Mar. 1998.

MELO,H. P. de. A pobreza e as políticas de gênero no Brasil. Unidade Mujer y Desarrollo - Projecto "Governabilidade democrática e igualdade de gênero". Santiago de Chile, junho, 2005.

MOGHADAM, V. The feminisation of poverty: notes on a concept and trend. Normal: Illinois StateUniversity, (Women'sStudiesOccasionalPaper, n. 2), 1997.

MOREIRA, R.B. Pobreza e desigualdade rural na região Sudeste sob o enfoque da pluriatividade e rendas não-agrícolas. Dissertação, $105 \mathrm{p}$. Universidade Federal de Viçosa, 2010.

NEDER, H.D..Os efeitos das atividades não agrícolas na distribuição de renda do meio rural do Brasil. Revista de Economia e Sociologia Rural, v. 41, n. 1, p. 265-290, 2003.

NOVELLINO, M. S. F. Os estudos sobre feminização da pobreza e políticas públicas para mulheres. In: Anais do XIV Encontro Nacional de Estudos Populacionais. Caxambu, v. 1, 2004.

OLIVEIRA, N. S. M. N. de. Pobreza das mulheres chefes de família da Região Nordeste do Brasil: uma análise multidimensional. 2018. 184 f. Tese (Pós-Graduação, Doutorado em Desenvolvimento Regional e Agronegócio) - Universidade Estadual do Oeste do Paraná, Toledo/Brasil, 2018.

PEARCE, D. The feminization of poverty: women, work and welfare. Urbanand Social ChangeReview, v. 11, p. 28-36, 1978.

PEREIRA, A. F. C.; LIMA, J. R. F.; JUSTO, W. R. Impactos das rendas não-agrícolas sobre os indicadores de pobreza Foster-Greer-Thorbecke (FGT) para as famílias rurais do estado de Pernambuco. In: IV Encontro Pernambucano de Economia, Recife, Pernambuco, 22 p., 2015.

PEREIRA, A. F. C. Impactos da pluriatividade e rendas não-agrícolas sobre a incidência de pobreza nas famílias agrícolas brasileiras. / Alan Francisco Carvalho Pereira. - 2017. 135f. :il.; $30 \mathrm{~cm}$.

RAVALLION, M.; BIDANI, B. How Robust Is a Poverty Profile? World Bank. EconomicReview 8 (1): 75-102, 1994.

SILVEIRA, E. C; SILVA, S. de F. M. Chefia feminina: uma análise sobre a estrutura das famílias monoparentais femininas e a feminização da pobreza. In: IV Seminário CETROS Neodesenvolvimentismo, Trabalho e Questão Social, 2013, Fortaleza. Anais... Fortaleza: UECE, 2013. 
SIQUEIRA, M. L.; SIQUEIRA, M. L. Desigualdade de renda no nordeste brasileiro: uma análise de decomposição. In: ENCONTRO REGIONAL DE ECONOMIA, 11, 2006, Fortaleza. Anais. Rio de Janeiro, Anpec,. P. 3 - 17., 2006.

WORLD BANK. Handbook on poverty and inequality. By Jonathan Henry Haughton,Shahidur R. Khandker. Document of the World Bank, Washington, DC, 2009.

WRIGHT, R. E. A feminisation of poverty in GreatBritain?Review of Income and Wealth, v. 38, n. 1, p. 17-25, Mar. 1992. 